\title{
Wavelets Application in Prediction of Friction Stir Welding Parameters of Alloy Joints from Vibroacoustic ANN-Based Model
}

\author{
Emilio Jiménez-Macías, ${ }^{1}$ Angel Sánchez-Roca, ${ }^{2}$ Hipólito Carvajal-Fals, ${ }^{2}$ \\ Julio Blanco-Fernández, ${ }^{3}$ and Eduardo Martínez-Cámara ${ }^{3}$ \\ ${ }^{1}$ Electrical Engineering Department, University of La Rioja, 26004 Logroño, Spain \\ ${ }^{2}$ Faculty of Mechanical Engineering, University of Oriente, 90900 Santiago de Cuba, Cuba \\ ${ }^{3}$ Mechanical Engineering Department, University of La Rioja, 26004 Logroño, Spain
}

Correspondence should be addressed to Emilio Jiménez-Macías; emilio.jimenez@unirioja.es

Received 1 March 2014; Accepted 23 March 2014; Published 18 May 2014

Academic Editor: Eugene B. Postnikov

Copyright (C) 2014 Emilio Jiménez-Macías et al. This is an open access article distributed under the Creative Commons Attribution License, which permits unrestricted use, distribution, and reproduction in any medium, provided the original work is properly cited.

\begin{abstract}
This paper analyses the correlation between the acoustic emission signals and the main parameters of friction stir welding process based on artificial neural networks (ANNs). The acoustic emission signals in $Z$ and $Y$ directions have been acquired by the $\mathrm{AE}$ instrument NI USB-9234. Statistical and temporal parameters of discomposed acoustic emission signals using Wavelet Transform have been used as input of the ANN. The outputs of the ANN model include the parameters of tool rotation speed and travel speed, and tool profile, as well as the tensile strength. A multilayer feed-forward neural network has been selected and trained, using Levenberg-Marquardt algorithm for different network architectures. Finally, an analysis of the comparison between the measured and the calculated data is presented. The model obtained can be used to model and develop an automatic control of the parameters of the process and mechanical properties of joint, based on the acoustic emission signals.
\end{abstract}

\section{Introduction}

Friction stir welding (FSW), invented by The Welding Institute of the UK in 1999, is an energy-efficient, environmentfriendly, and versatile joining technique that has proved to be one of the most significant achievements in the field of joining aluminium alloys [1].

The principal advantage of this technology consists in the possibility of being used to successfully join materials that are difficult-to-weld or unweldable by fusion welding methods [2]. FSW has been widely used in the areas of space, aircraft, marine, fuel tank, and food-saving industry [3].

Science and technology development has contributed to obtain models with similar behaviour to the physical process in the aspects into consideration, which allow avoiding the complexity of the studied phenomenon. Based on this and the growing acceptance of friction stir welding as a reliable welding technique, it is necessary to increase the development of different online monitoring methods for modelling and control of the quality of the welds [4].

The monitoring of acoustic emission (AE) $[5,6]$ signals is a nondestructive evaluation technique for real time applications. It is characterized by a more precise representation of the behaviour of the phenomenon, but its high complexity must be considered in the analysis. Different works reported [7-11] show the application of nondestructive techniques (NDT) on the mechanical and manufacturing processes.

Roca et al. [9] and Macías et al. [10] demonstrated the advantage of the application of $\mathrm{AE}$ as a nondestructive method for characterization of stability in gas metal arc welding process. A new index for the stability analysis of GMAW processes taking as a basis the AE generated by the arc was obtained in this research. The authors confirmed the effectiveness of the method proposed as a new way for the analysis of stability in welding process. 
Fernández et al. [11] demonstrated the feasibility of the application of AE for monitoring the FSW systems. The authors presented a method to evaluate the effect of the tool profile on friction stir welding process in the aluminium AA 1050, using acoustic emission signals.

Soundararajan et al. [7] determined the correlation between the losses of contact of the tool with the workpiece based on features of the AE signal, using the Fast Fourier Transform (FFT), the Short-Time Fourier Transform (STFT), and the Discrete Wavelet Transform (DWT). The authors investigated the possibility of applying $\mathrm{AE}$ techniques for the in-process monitoring of the FSW process. They also demonstrated that $\mathrm{AE}$ signal in the frequency domain is sensitive to the change in the depth of penetration of the tool.

The applicability of vibroacoustic signals in the analysis of FSW process was confirmed by Suresha et al. [8]. These authors developed a conceptual model to correlate the FSW process parameters, tool profile, material flow pattern, microstructures, and mechanical properties.

Welding processes are physically complex in nature, nonlinear, and dynamic, presenting a large number of input parameters; there is no close mathematical model which can describe the behaviour of these processes [7]. On the other hand, in the last years, artificial intelligence techniques and especially artificial neural networks (ANNs) are being used as a tool to model different complex processes.

ANNs can be used in many applications for process modelling, monitoring, and control purposes, using wavelets [12-15]. They also can be used to estimate parameters that usually cannot be measured on-line. In the fields of biology, electronics, computer science, mathematics, and engineering, $\mathrm{ANN}$ is one of the most important research areas [16, 17].

ANN possesses an excellent ability to learn and generalise (interpolate) complicated relationships between input and output variables of such type of systems. In recent times, in the engineering area, the computer-aided ANN modelling is becoming more and more important. Various developments of ANN applied to processes are reported in the literature $[2,16,18]$.

Okuyucu et al. [3] and others authors [16, 19] used a ANNbased model applied to FSW of aluminium alloy in order to predict the mechanical properties of welding using the process parameters as input of the model.

Lakshminarayanan and Balasubramanian [19] performed a comparison between response-surface methodology and artificial neural network models to predict the tensile strength of FSW AA7039 aluminium alloy. The authors used, as input of the model, process parameters such as tool rotational speed, welding speed, and axial force.

Tansel et al. [18] carried out a study, using ANN, to estimate the optimal operating condition of the FSW process. The authors used five separate ANNs as an excellent optimization tool for FSW, in order to obtain the relationship between two identical input parameters and each one of the characteristics of the welding zone.

All models based on ANN previously obtained for FSW are based on correlating the mechanical properties of the joint (tensile strength, hardness, etc.) $[7,8,11]$ obtained from the mechanical tests and microstructural characteristics based on process parameters (tool rotation speed and travel speed) predefined.

In the existing literature, it is not possible to find a model that predicts the values of the parameters of the process and the mechanical properties applying a noninvasive technique such as EA. The new model shown in this paper aims to fill this lack by providing a detailed explanation of the methodology and results obtained from processing vibroacoustics signals and developing a model based on ANN. These results also suggest the possibility of developing an acoustic emission-based model for monitoring and controlling changes on the parameters of FSW processes and predicting mechanical properties of joint.

\section{Experimental}

The plates used in the present paper are aluminium AA1050 $\mathrm{H} 24$ with dimensions $200 \mathrm{~mm}(L), 100 \mathrm{~mm}(W)$, and $3 \mathrm{~mm}$ $(T)$. This alloy is known for its electrical conductivity and it is commonly used in automotive trim, chemical and food industry equipment, light reflectors, and strips for heat exchangers. The chemical compositions (wt\%) of AA1050 $\mathrm{H} 24$ used for experimentation were $0.25 \mathrm{Si}, 0.40 \mathrm{Fe}, 0.05 \mathrm{Cu}$, 0.05 Mn, 0.05 Mg, 0.07 Zn, 0.05 Ti, 0.03 others, and 99.05 Al.

The FSW process was carried out using a CNC machine with displacement control [11]. The plates were butt-joined by FSW and locked in position using mechanical clamps. The travel angle used was $0^{\circ}$ and the direction of welding was normal to the rolling direction. The experiments were performed according to the experimental setup shown in Figure 1.

A large number of trial runs were performed to select the practical working limits of FSW process parameters. To implement this study, the obtaining of defect-free joints inside the experimental region was verified. The tool life was another factor taken into account. It was ensured that the tool maintained its dimensions during the performance of the trials.

During the test, three levels of the travel speed (40, 63, and $100 \mathrm{~mm} \cdot \mathrm{min}^{-1}$ ) and tool rotation speed (450, 710, and $1120 \mathrm{rpm}$ ) were used (two limits and a central level). Experimental designs of factorial type were also used (18 welding experiments developed in total). To reduce the experimental error, all the welding experiments were performed three times under the same conditions.

The diameters of the tool probe and tool shoulder were 3 and $10 \mathrm{~mm}$, respectively. Two different pin profiles were considered: a conventional cylindrical pin with grooves (Tool 1) and a threaded cylindrical pin (Tool 2). The shoulder profile for these tools was concave.

The vibroacoustic signals generated during the welding process in $Z$ and $Y$ directions (Figure 1) were captured and recorded using the AE instrument NI USB-9234 connected to $\mathrm{PC}$. The AE signals generated as a result of the external noise were monitored before welding.

All signals were sampled at $51.2 \mathrm{kHz}$ and processed using MATLAB software. Two acoustic emission sensors (piezoelectric-based) were arranged perpendicularly on 


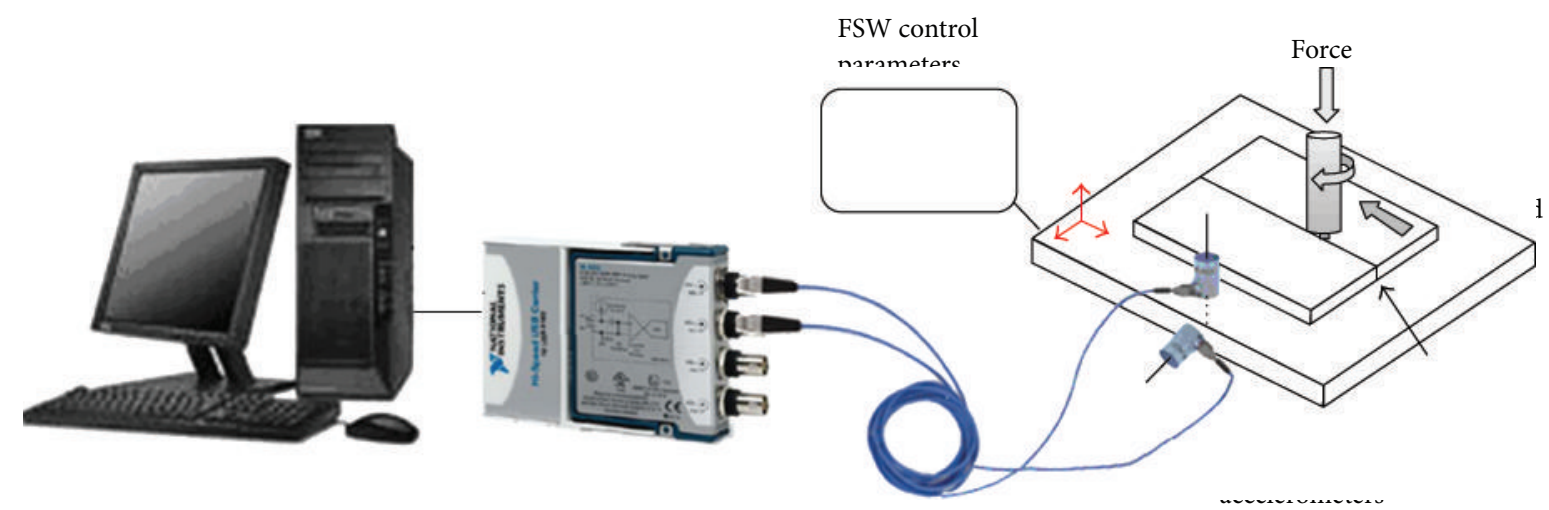

FIGURE 1: Schematic diagram of the experimental setup.

the top and lateral surface of the backing plate. The sensors were placed on the backing plate and coupling was used in order to provide a good acoustic path between the backing plate and the sensor.

2.1. Extract Features of Vibroacoustic Signals. A critical task of the AE signal processing is the selection of an appropriate frequency range to provide information about the application process behaviour. It is necessary to carry out an additional statistical analysis of the information in order to obtain the adequate characterisation of the acquired data.

In this piece of research, the statistical parameters of vibroacoustic signals and their Wavelet Transform (WT) [20, 21] were used in order to obtain the data input of the ANN used. The mean value, standard deviation, and coefficient of variation are the most generally used indices for studying welding process.

WT has settled as an effective tool to decompose nonstationary signals such as vibroacoustic signals. WT allows the use of long time intervals where more precise low frequency information is required, and shorter regions where highfrequency information is needed. In this paper, Daubechies wavelets of order eight have been used to decompose the signals at different scales in order to obtain the specific frequency bands that are useless for the analysis.

In this case, the application of WT is equivalent to the result of passing the vibroacoustic signals through a filter with resulting frequency bandwidths $[0,100 \mathrm{~Hz}],[100,200 \mathrm{~Hz}]$, [200, $400 \mathrm{~Hz}],[400,800 \mathrm{~Hz}],[800 \mathrm{~Hz}, 1.6 \mathrm{kHz}],[1.6,3.2 \mathrm{kHz}$, $[3.2,6.4 \mathrm{kHz}],[6.4,12.8 \mathrm{kHz}]$, and $[12.8,25.6 \mathrm{kHz}]$. This range of frequency band was associated with the order (eight) of the WT.

Chen et al. [22] demonstrated the validity of WT analysis of acoustic emission in monitoring FSW of 6061 aluminium. The authors showed the occurrence of significant sudden changes in the band energy at the moment when the probe penetrates into and pulls out of the weld joint, as well as when the shoulder makes contact with or detaches from the plates.

Each one of the obtained signals was characterized statistically in the different energy bands as a result of the WT decomposition. Finally, a significance testing was carried out with the objective of extracting the statistical parameters that showed significant differences to use them as input to the ANN.

2.2. Artificial Neural Network Methodology. The methodology presented below has been used to obtain conclusions about the effectiveness of ANN to evaluate changes in parameters process and mechanical properties based on relationship between FSW parameters and vibroacoustic signals generated by process.

A neural network, which can capture and represent the relationship between the vibroacoustic signals, mechanical properties, and process parameters, has been developed in this stage. Multilayered perception (MLP) neural network was one of the most popular and earliest developed network architecture for engineering applications, and it is commonly used to solve difficult predictive modelling problems.

The structure of a neural network is determined by the number of layers and the number of nodes in each layer. In this paper, a back propagation (BP) type network has been selected, specifically a standard BP network composed of two layers.

Equation (1) shows the output function of the ANN used, where $W_{1}$ and $W_{2}$ represent the matrix of the weight of input hidden neurons and hidden output neurons, respectively, $b_{1}$ and $b_{2}$ represent the biases of each one of the neurons of the hidden layer and the output layer, and $x$ represents the input vector to the network. Consider

$$
y=f\left(W_{2} f\left(W_{1} x+b_{1}\right)+b_{2}\right) .
$$

The system has three layers, which are input, hidden, and output layers, respectively. The input layer consists of a number of input nodes called neurons and collects all the input factors. In this case, it corresponds to the features of vibroacoustic signals. The established input values were denoted as $x_{1}$ to $x_{n}$.

Information from the input layer is then processed in the course of one hidden layer. Each neuron in the hidden layer is connected to every neuron in the previous layer via a set of weighted links $\left(W_{1}\right)$. This weighted sum is passed through a nonlinear activation function to produce an output vector $(y)$ in the range of 0 to 1 . Precisely, the MLP is ideal for modelling nonlinear relationships, such as this one between 
the generated vibroacoustic signals and the FSW parameters. The transfer functions for all hidden neurons are tangent sigmoid functions, as shown in

$$
f(x)=\frac{\exp (x)-\exp (-x)}{\exp (x)+\exp (-x)}
$$

FSW parameters are computed in the output layer, which contains a neuron for each parameter that is to be modelled. Again, weights are used to connect each output neuron to every neuron in the previous hidden layer $\left(W_{2}\right)$. The neurons in the hidden layers are computational units that perform nonlinear mapping between inputs and outputs. The output value of the ANN $(y)$ is in correspondence with the established welding parameters. The transfer functions for the output neurons are linear functions, as shown in

$$
f(x)=x .
$$

Determining the number of hidden neurons is critical in the design of neural networks. An overabundance of hidden neurons gives too much flexibility, which usually leads to over fitting. On the other hand, too few hidden neurons restrict the learning capability of a network and degrade its approximation performance.

ANN learns from examples, namely, patterns. In order to introduce the patterns to the ANN, it has been necessary to standardise them by using a preprocessing stage. To train and test a neural network, input data and corresponding target values are necessary. A total of 126 input-output data patterns have been used into a training set and 18 into a testing set.

2.3. Artificial Neural Network Training Algorithms. The neural network was trained using two different training algorithms:

(1) gradient descent with momentum algorithm,

(2) Levenberg-Marquardt algorithm.

BP learning uses a gradient descent algorithm to minimise the mean square error between the target data and the predictions of a neural network. One of the major problems with the basic BP algorithm (gradient descent algorithm) has been the extended training time required. The technique for accelerating convergence falls into two main categories: heuristic methods and standard numerical optimisation methods, such as the Levenberg-Marquardt BP (LMBP) algorithm.

The LMBP algorithm is similar to the quasi-Newton method, in which a simplified form of the Hessian matrix (second derivatives) is used. When the cost function has the form of a sum of squares, then the Hessian matrix $H$ can be approximated as

$$
H=J^{T} J
$$

and the gradient $g$ can be computed as

$$
g=J^{T} e,
$$

where $J$ is the Jacobian matrix that contains the first derivatives of the network errors with respect to the weights and biases and $e$ is a vector of network errors. The Jacobian matrix can be computed through a standard back-propagation technique that is much less complex than computing the Hessian matrix. The LMBP algorithm is the fastest algorithm that has been tested for training multilayer networks of moderate size. Equation (6) shows the function used during the training of ANN:

$$
F(w)=\frac{1}{2} e^{T}(w) \cdot e(w)
$$

The performances of these two training algorithms are compared to decide which algorithm performs better than the other one. After examining the working of different architectures of networks, the test data was introduced into the ANN to evaluate the performance obtained. Figure 2 shows the general steps of the methodology used in this piece of research.

The preprocessing stage develops the transformation of the input or target data before it is presented to the neural network. On the other hand, the postprocessing stage converts normalized outputs back into the same units that were used for the original targets.

Based on the "intelligence" of the new model obtained, it is possible to predict values of process parameters not shown during the experiments based on vibroacoustic signal. The creation of this model will allow the development of future applications for monitoring and automatic control of FSW process parameters based on the measurement of vibroacoustic signals generated by the process.

\section{Results and Discussion}

Acoustic emission is a nondestructive evaluation technique for real time monitoring applications. In this piece of research, all the vibroacoustic signals have been characterized. The key to the ANN modelling was to extract the features of the vibroacoustic signals generated by the FSW process in order to correlate them with the process parameters and mechanical properties of FSW joint.

Figures 3(a) and 3(c) show the cross-section perpendicular to the welding direction for two representative conditions with tool rotation speed $=710 \mathrm{rpm}$, travel speed $=$ $40 \mathrm{~mm} \cdot \mathrm{min}^{-1}$ (Figure 3(a), condition 1), and travel speed $=$ $63 \mathrm{~mm} \cdot \mathrm{min}^{-1}$ (Figure 3(c), condition 2).

In Figures 3(a) and 3(c), different zones in the weld can be appreciated, such as nugget zone (NZ), thermo mechanically affected zone (TMAZ), and heat affected zone (HAZ). Figure 3 allows observing the good quality of the joints. The macroimages of the weld zone presented in Figures 3(a) and 3(b) show no weld defects and macrostructural continuity.

3.1. Vibroacoustic Signals Features Extraction. Noise removal constituted the initial stage of characterizing AE signals, producing, as indicated in the previous section, the results shown in preceding works [11,23]. 


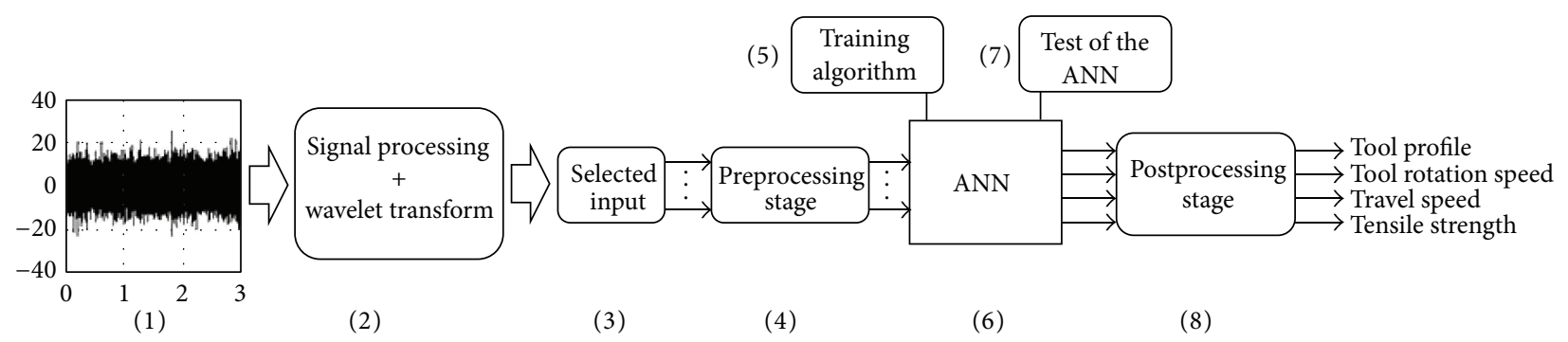

FIgUre 2: Methodology of ANN development.

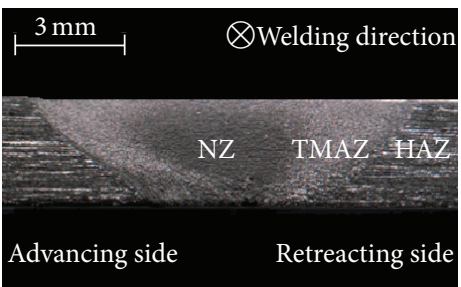

(a)

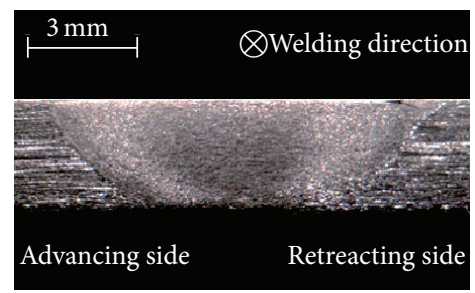

(c)

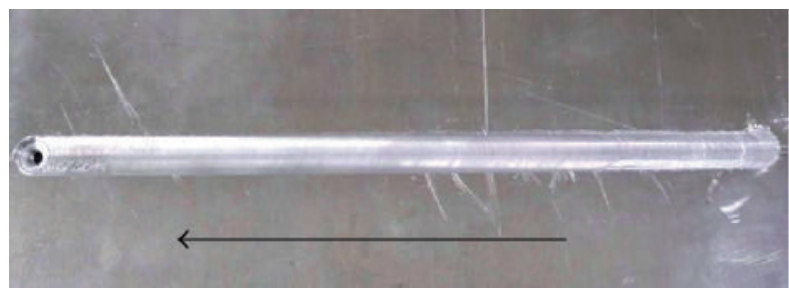

(b)

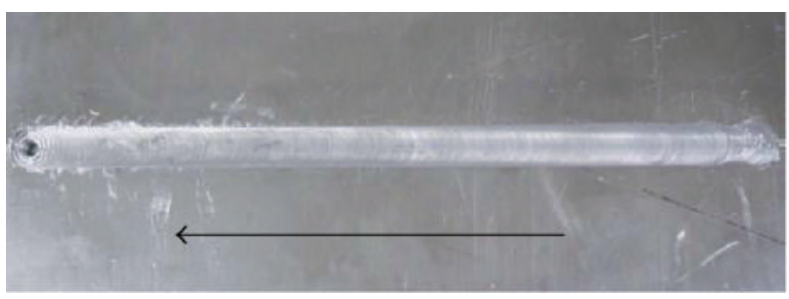

(d)

FIgURE 3: Macrograph of two evaluated conditions. (a-c) Cross-sectional weld zone; (b-d) longitudinal weld zone.

The extraction of features of the vibroacoustic signals was based on statistical characterization. Several methods are useful in determining which features of these signals are used as input of ANN. In this paper, the analysis of variance (ANOVA) is a method used to investigate which statistical parameters of the signals are significantly affected by changes in welding parameters (outputs of the ANN).

Figure 4 shows the graphics of the vibroacoustic signals corresponding to conditions 1 and 2. These conditions present the same tool rotation speed $=710 \mathrm{rpm}$ and the same tools profile, in this case a cylindrical probe with grooves. The travel speeds were $40 \mathrm{~mm} \cdot \mathrm{min}^{-1}$ (Figure $4(\mathrm{a})$ ) and $63 \mathrm{~mm} \cdot \mathrm{min}^{-1}$ (Figure 4(b)), respectively.

As it can be appreciated in Figure 4, the amplitude of the vibroacoustic signal is higher for condition 1 , caused by the smaller travel speed $\left(40 \mathrm{~mm} \cdot \mathrm{min}^{-1}\right)$. The result of the application of ANOVA at all vibroacoustic signals shows that only two features were influenced by changes on the parameter levels. In this case, the data median and RMS were selected as input of the ANN. Table 1 shows the values of the statistical parameters for the analysed signals showed in Figure 4.

Considering the complex nature of the vibroacoustic signals generated by the FSW process, the use of only two vibroacoustic features is not sufficient to obtain a relationship
TABLE 1: Statistical characterization of the vibroacoustic signals.

\begin{tabular}{lcc}
\hline Condition & RMS & Median \\
\hline Condition 1 & 5.71 & 0.096 \\
Condition 2 & 4.00 & 0.0093 \\
\hline
\end{tabular}

between the vibroacoustic signals and the process parameters. Therefore, the present study considers the inclusion of additional features of the vibroacoustic signals based on the time-frequency properties, using a WT to obtain additional statistical features related to different frequency band of the signals.

3.2. Wavelet Transform for New Features. The DWT has been utilized in this work to obtain additional statistical features of each series of decomposed vibroacoustic signals.

The result of the DWT is a series of decomposed signals belonging to different frequency bands. The graphics in Figures 5(a) and 5(b) represent the WT of the vibroacoustic signal for condition 1 and condition 2, respectively. In this study, the DWT decomposition is applied using eight levels and mother wavelet Daubechies (Db5).

In Figure 5, all decomposed frequency bands for the vibroacoustic signals corresponding to condition 1 and condition 2 can be appreciated. A visual inspection of these figures 


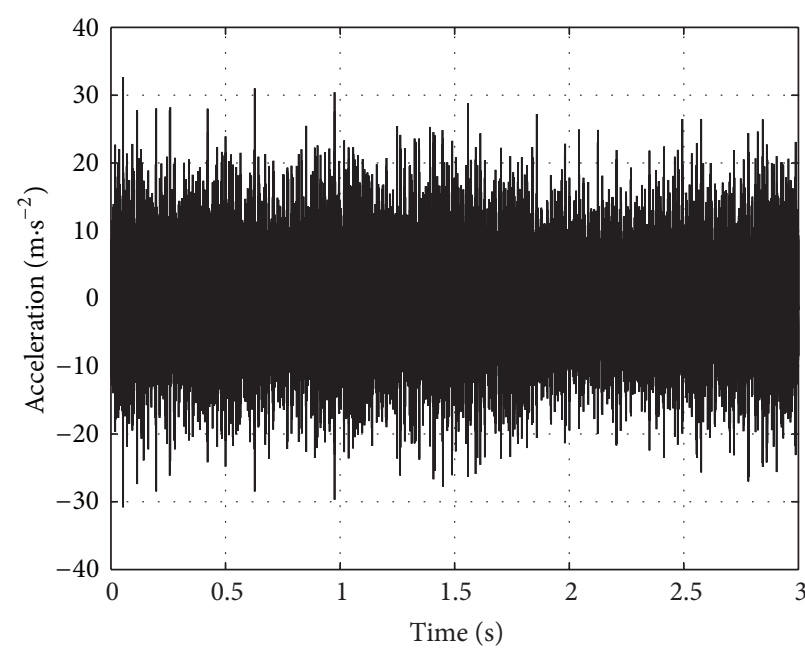

(a)

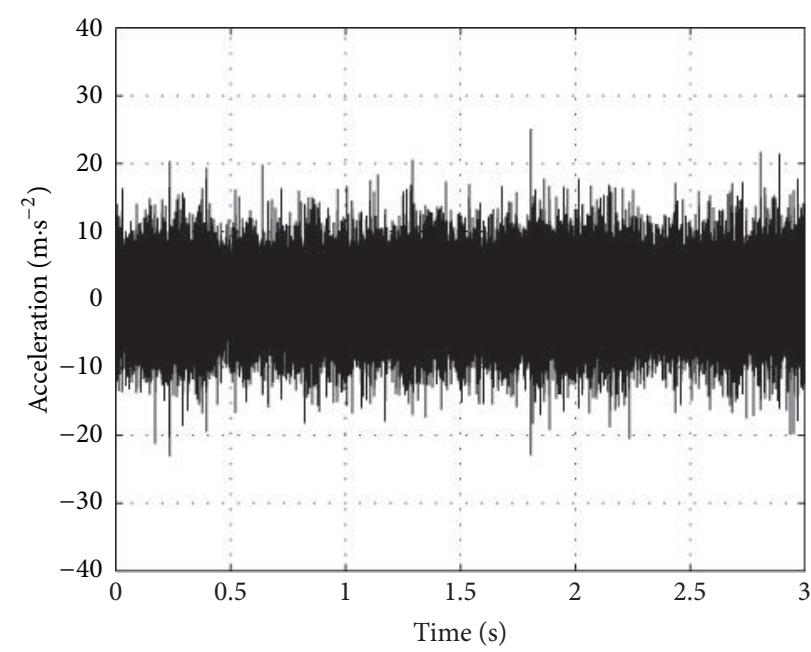

(b)

FIGURE 4: Vibroacoustic signals for two welding conditions. (a) Condition 1; (b) condition 2.

enables observing the differences between the signals for each similar frequency band.

The $F$-test has been used to determine which vibroacoustic features were affected by changes on the FSW parameters. For the analyses, the bands of frequency associated with the noise that were demonstrated unnecessary in the previous epigraph were not taken into account.

Figure 6 shows the results of ANOVA methods for three selected features, extracted from the DWT decomposition, used as input of the ANN for each one of the welding parameters.

As result of the ANOVA statistical analysis of each decomposed signal, 14 new features were selected as input of the ANN. The defined inputs exhibit means of groups significantly different due to changes on the levels of the process parameters.

3.3. Neural Network. In recent years, ANNs have been applied to a wide range of fields and have provided solutions for scientific and industrial problems. This paragraph shows the features of vibroacoustic signals selected as input of the ANN with highly significant difference. The results of the selected topology and the training algorithm are also presented.

In order to reduce the number of input (14 inputs) to ANN, the sensitivity and principal component analysis was developed. This procedure reduces the dimension of the input vectors eliminating redundant components. Finally, a total of 10 feature values $\left(x_{1}, \ldots, x_{10}\right)$ have been selected: two extracted from the time domain signals and eight statistical parameters based on DWT decomposed signals. In this case, the vibroacoustic features selected as input of the ANN are shown in Table 2. These features show a significant incidence with the variations on the welding process parameters and tensile strength.

Table 3 presents three options of neural network architecture tested. The evaluation of different architectures
TABLE 2: Selected input of ANN.

\begin{tabular}{lcc}
\hline Factor & Vibroacoustic parameter & Symbol \\
\hline$x_{1}$ & Median of vibroacoustic signal & $M_{V}$ \\
$x_{2}$ & RMS of vibroacoustic signal & $\mathrm{RMS}_{V}$ \\
$x_{3}$ & Median of $A 8$ DWT & $M_{A 8}$ \\
$x_{4}$ & RMS of $A 8$ DWT & $\mathrm{RMS}_{A 8}$ \\
$x_{5}$ & Median of D8 DWT & $M_{D 8}$ \\
$x_{6}$ & RMS of D8 DWT & $\mathrm{RMS}_{D 8}$ \\
$x_{7}$ & RMS of D7 DWT & $\mathrm{RMS}_{D 7}$ \\
$x_{8}$ & RMS of D5 DWT & $\mathrm{RMS}_{D 5}$ \\
$x_{9}$ & RMS of D4 DWT & $\mathrm{RMS}_{D 4}$ \\
$x_{10}$ & Median of D1 DWT & $M_{D 1}$ \\
\hline
\end{tabular}

TABLE 3: Topology of tested networks.

\begin{tabular}{lccc}
\hline $\begin{array}{l}\text { Topology } \\
\text { (input-hidden-output) }\end{array}$ & $\begin{array}{c}\text { Mean square } \\
\text { error }\end{array}$ & $\begin{array}{c}\text { Number of } \\
\text { training } \\
\text { passes }\end{array}$ & $\begin{array}{c}\text { \% Correct } \\
\text { evaluation }\end{array}$ \\
\hline $10-10-3$ & 0.0917 & 12 & 98.24 \\
$\mathbf{1 0 - 1 6 - 3}$ & $\mathbf{0 . 0 9 9 8}$ & $\mathbf{6}$ & $\mathbf{9 9 . 9}$ \\
$10-22-3$ & 0.0991 & 8 & 99.1 \\
\hline
\end{tabular}

and training algorithm demonstrated that a network with one hidden layer (16 neurons in hidden layer) trained by Levenberg-Marquardt algorithm shows good performance and provides the smaller final errors.

The selected network architecture consists of 10 input neurons, 16 hidden neurons with nonlinear activation function (log sigmoid), and three output neurons (tool rotation speed, travel speed, and tool profile) with linear activation function. The topology of the selected network 10-16-3 is shown in Figure 7.

The ANN with smaller number of nodes in the hidden layer was not converging and the pattern error value 

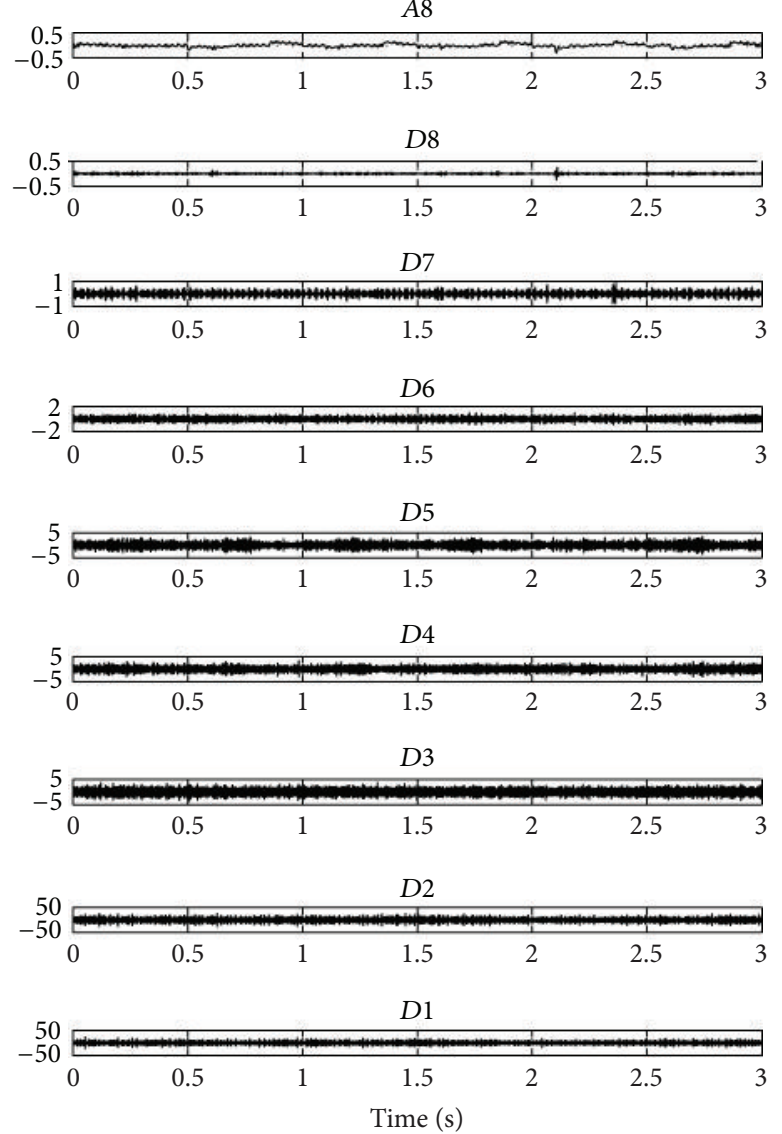

(a)

FIGURE 5: Wavelet Transforms.

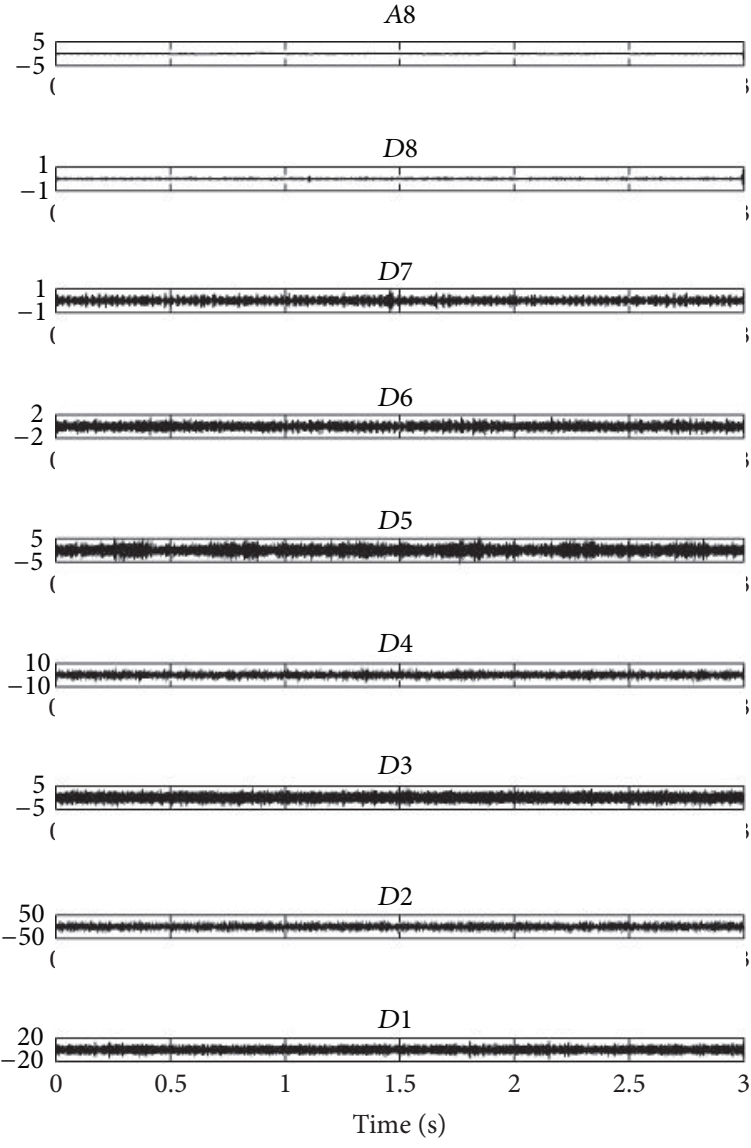

(b) remained high. With architecture 10-16-3, the final correct evaluation obtained was $99.9 \%$. With this weight matrix, correct results could be obtained with a confidence of $95 \%$. The use of more than the optimal number of hidden layers in the network may result in undesirable overfitting of the data.

The transfer function log sigmoid used in this study is given by

$$
Y_{i}=\frac{1}{1+e^{-x_{i}}}
$$

The final operation of the network is governed by

Tool profile $=N_{1} W_{1,1}^{2}+N_{2} W_{1,2}^{2}+N_{3} W_{1,3}^{2}+N_{4} W_{1,4}^{2}$

$$
+\cdots+N_{16} W_{1,16}^{2}+b_{1,1}^{2} \text {, }
$$

Tool rotation speed $=N_{1} W_{2,1}^{2}+N_{2} W_{2,2}^{2}+N_{3} W_{2,3}^{2}+N_{4} W_{2,4}^{2}$

$$
+\cdots+N_{16} W_{2,16}^{2}+b_{2,1}^{2}
$$

Travel speed $=N_{1} W_{3,1}^{2}+N_{2} W_{3,2}^{2}+N_{3} W_{3,3}^{2}+N_{4} W_{3,4}^{2}$

$$
+\cdots+N_{16} W_{3,16}^{2}+b_{3,1}^{2} \text {, }
$$

Tensile strength $=N_{1} W_{4,1}+N_{2} W_{4,2}+N_{3} W_{4,3}+N_{4} W_{4,4}$

$$
+\ldots+N_{16} W_{4,16}+b_{4,16}
$$

where $N_{i}=f\left(W_{1} \cdot x+b_{1}\right), i=1,2,3,4, \ldots, 16$.

The AE-based model governed by a new mathematical equation system (8) improves the results of the existing models, and furthermore it uses a noninvasive technique (specifically AE) to predict the mechanical properties of joint and the process parameters. The proposed model can estimate the values of process parameters not determined during the experimental research.

3.4. Neural Network Evaluation. The testing of the neural network model was developed using new test sets to validate the generalization capability of the network. Once obtained the values of the weights and biases, they have been substituted in (8), which govern the operation of the ANN. After being trained by the training data set, the networks are checked to assure their ability to obtain the FSW process parameters and tensile strength based on the data test.

The obtained output values of the ANN were introduced into the postprocessing stage in order to obtain the real value of the process parameters. Figure 8 shows a comparison 


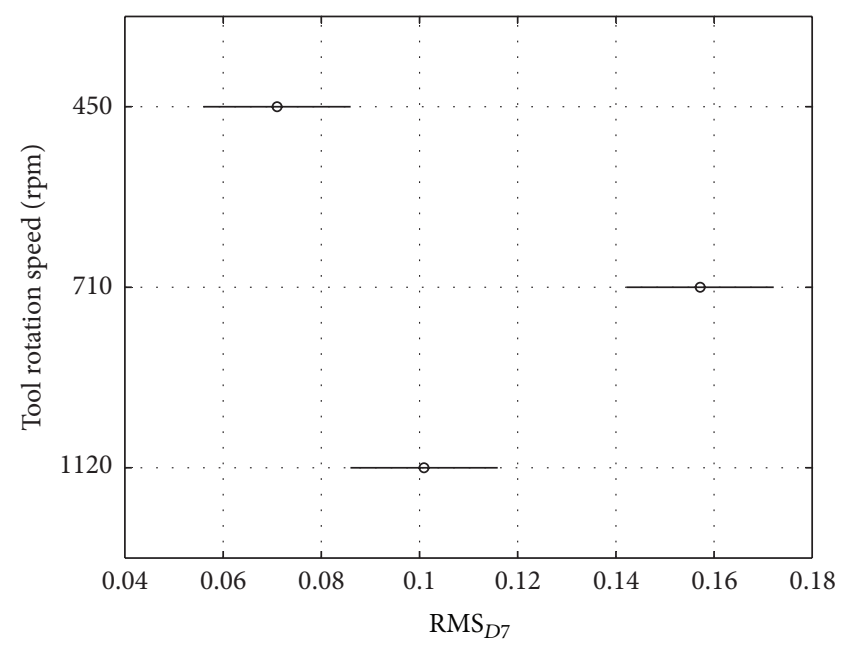

(a)

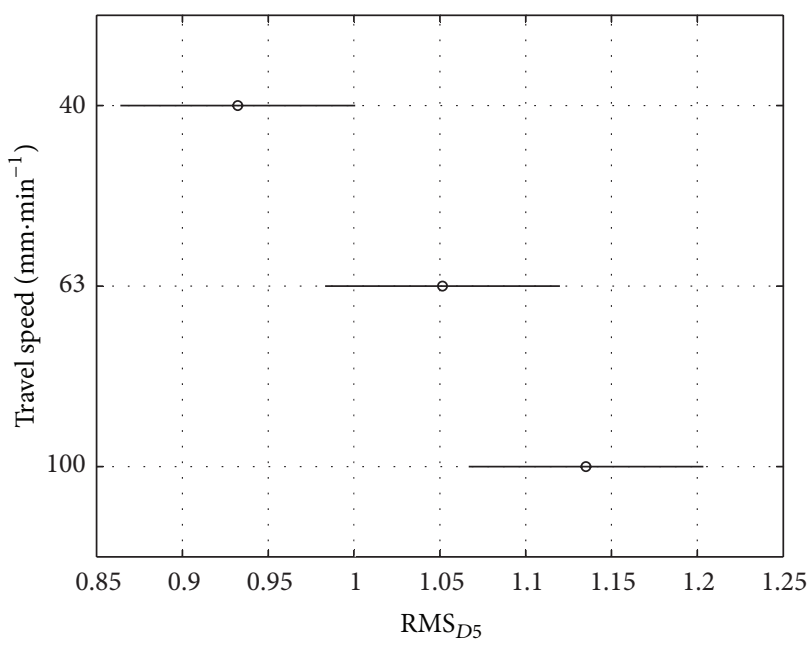

(b)

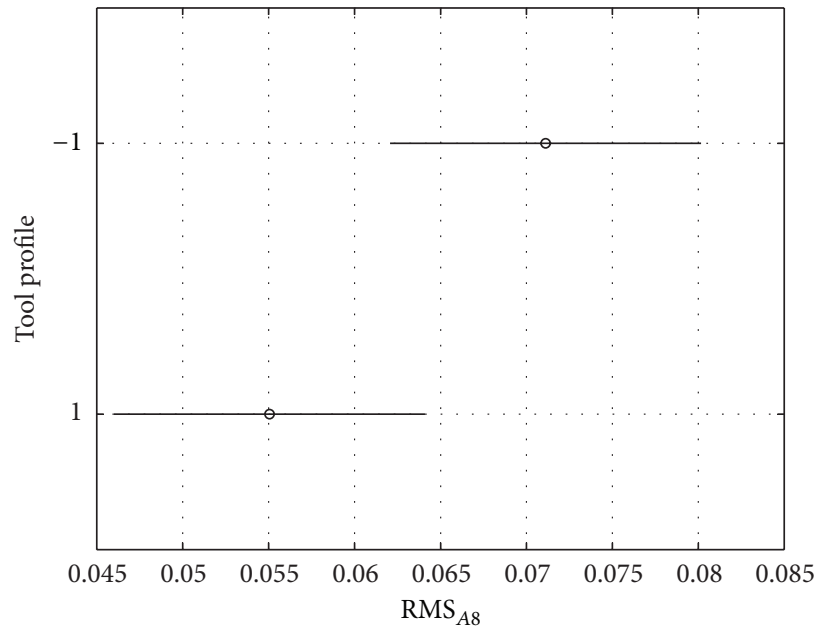

(c)

Figure 6: Influence of the FSW parameters on DWT decomposed signal. (a) Tool rotation speed, (b) travel speed, and (c) tool profile.

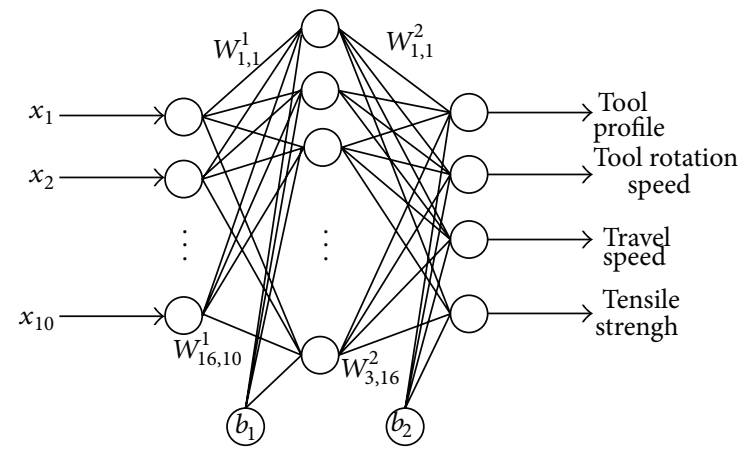

FIGURE 7: Topology of ANN.

between the process parameters and mechanical property of the FSW joint and values calculated using the ANN. The obtained correlation factor is also shown as a result of the regression analysis.
As it can be appreciated in Figure 8, the measured and predicted output values were close to each other. The evaluation of the ANN using the test data shows that a practically lineal relationship exists between the FSW parameters and the parameters calculated with the ANN by (1).

Figure 8(c) shows the comparative analysis between the tensile strength obtained from the ANN-based model and the one determined using the mathematical model developed in previous works [23]. In this case, the value of the adjusted coefficient of determination $r^{2}$ was $91.4 \%$ for mathematical model, indicating that less than $9 \%$ of the total variations are not explained by the model [22]. The neural network model predicts the resistance to traction in $99 \%$ of the cases.

The great advantage of obtaining the process parameters by using the ANN lies in the possibility of estimating more parameter values from the vibroacoustic signals, due especially to the generalization capability of the ANN.

Table 4 shows the results of the data predicted by the ANN for the two conditions, compared with the real data. 


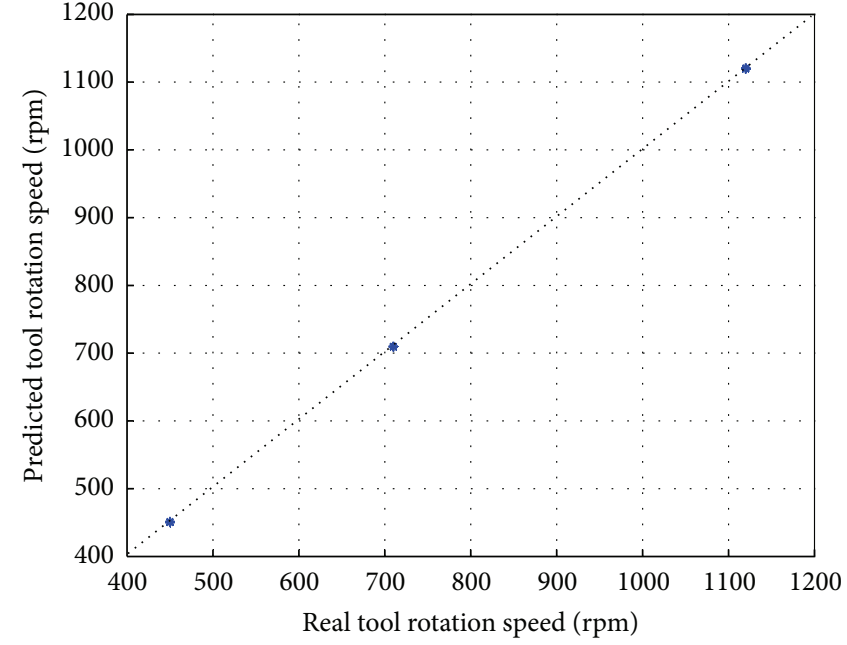

(a)

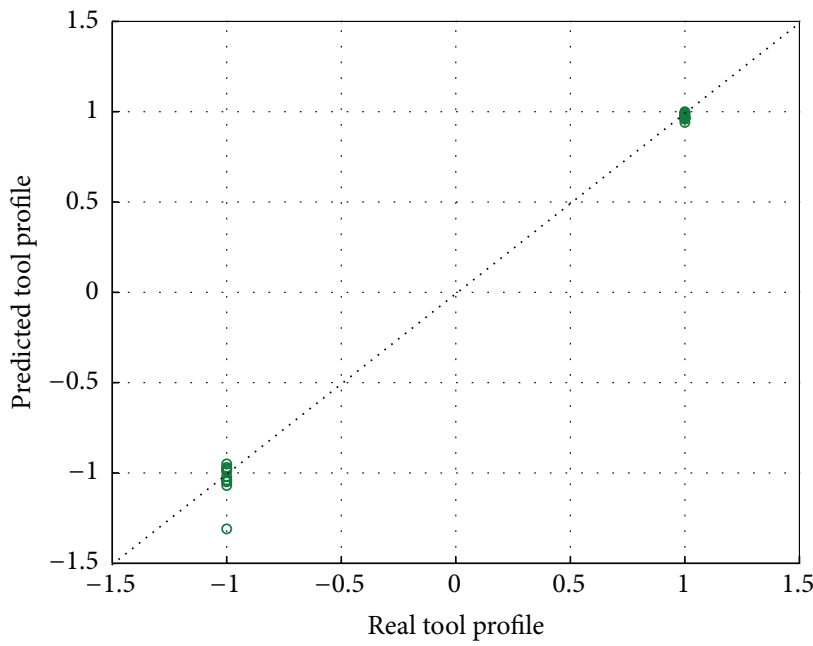

(c)

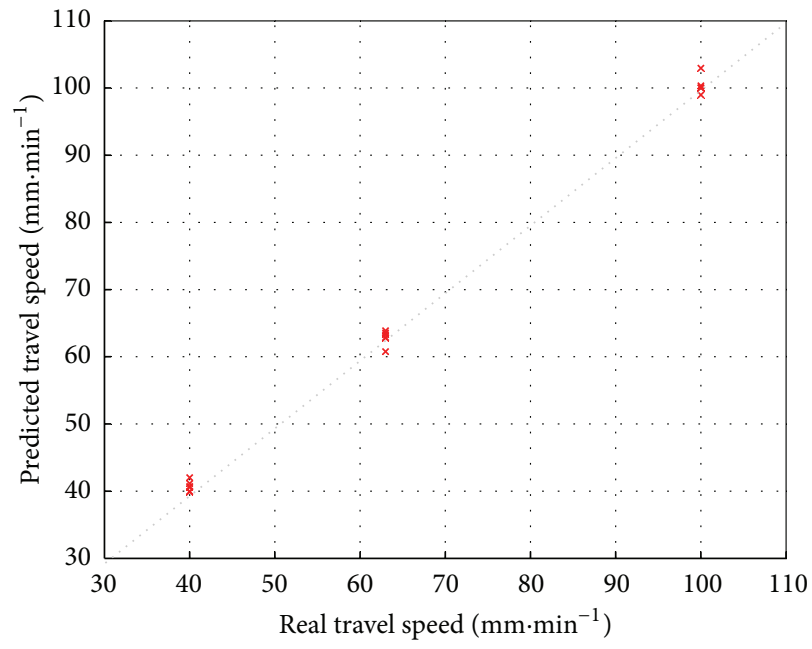

(b)

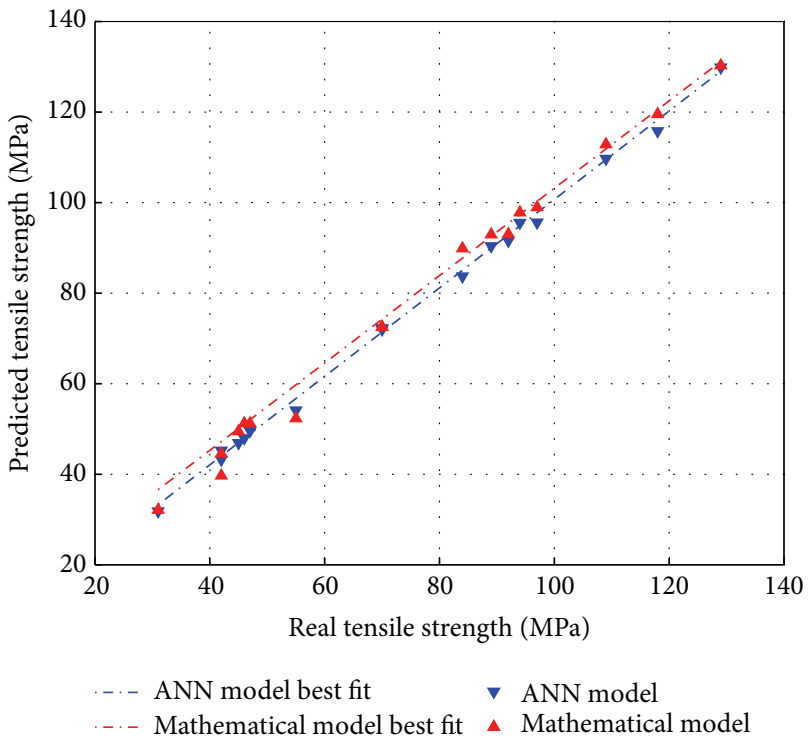

(d)

FIGURE 8: Predicted and measured process parameters. (a) Tool rotation speed, (b) travel speed, (c) tool profile, and (d) tensile strength.

The obtained results demonstrate the validity of the use of the ANN for the modelling of the FSW process parameters.

\section{Conclusions}

This paper evaluates the possibility of the use of ANN to predict the FSW process parameters by using the vibroacoustic signals generated by the process. It has been concluded that

(i) vibroacoustic signals processing, based on statistical characterisation in a specific range of frequencies, provides useful on-line information about the changes on the FSW process parameters and mechanical property of joint;

(ii) the results show that the new model obtained, based on ANN, is an effective technique to predict FSW process parameters and tensile strength of joint based on vibroacoustic signals features evaluation. The process parameters have been correctly identified in approximately $99 \%$ of the cases;

(iii) a new nondestructive modelling method is available to estimate welding parameters and mechanical properties, without interfering with the operation during production;

(iv) the Levenberg-Marquardt algorithm shows superior performance than gradient descent with momentum in this case;

(v) the effectiveness of the use of ANN to model complex relationships, parameters, and responses has been demonstrated, based on the statistical characterisation of the vibroacoustic signals; 
TABLE 4: Parameters calculated versus predicted.

\begin{tabular}{lcccc}
\hline Condition & $\begin{array}{c}\text { Tool rotation speed } \\
\text { (real/predicted) }\end{array}$ & $\begin{array}{c}\text { Travel speed } \\
\text { (real/predicted) }\end{array}$ & $\begin{array}{c}\text { Tool profile } \\
\text { (real/predicted) }\end{array}$ & $\begin{array}{c}\text { Tensile strength } \\
\text { (real/predicted) }\end{array}$ \\
\hline 1 & $710 / 709.94$ & $40 / 39.88$ & $1 / 1$ & $84 / 83.71$ \\
2 & $710 / 709.96$ & $63 / 63.89$ & $1 / 0.97$ & $70 / 71.96$ \\
\hline
\end{tabular}

(vi) The RMS of vibroacoustic signals is one of the key factors influenced by the process parameters;

(vii) wavelet-based analysis techniques can be used as a tool to model FSW process parameters.

\section{Conflict of Interests}

The authors declare that there is no conflict of interests regarding the publication of this paper.

\section{Acknowledgment}

This paper has been partially supported by the Project of the Spanish Government "DPI2011-25007. Friction Stir Welding of dissimilar materials. Characterization by acoustic emission techniques and artificial intelligence."

\section{References}

[1] G. M. Xie, Z. Y. Ma, and L. Geng, "Development of a finegrained microstructure and the properties of a nugget zone in friction stir welded pure copper," Scripta Materialia, vol. 57, no. 2, pp. 73-76, 2007.

[2] G. Buffa, L. Fratini, and F. Micari, "A neural network based approach for the design of FSW processes," Key Engineering Materials, vol. 410-411, pp. 413-420, 2009.

[3] H. Okuyucu, A. Kurt, and E. Arcaklioglu, "Artificial neural network application to the friction stir welding of aluminum plates," Materials and Design, vol. 28, no. 1, pp. 78-84, 2007.

[4] T. Jene, G. Dobmann, G. Wagner, and D. Eifler, "Monitoring of the friction stir welding process to describe parameter effects on joint quality," Welding in the World, vol. 52, no. 9-10, pp. 47-53, 2008.

[5] E. Castillo, D. P. Morales, A. García, F. Martínez-Martí, L. Parrilla, and A. J. Palma, "Noise suppression in ECG signals through efficient one-step wavelet processing techniques," Journal of Applied Mathematics, vol. 2013, Article ID 763903, 13 pages, 2013.

[6] R. Li and Y. M. Liu, "Wavelet optimal estimations for density functions under severely ill-posed noises," Abstract and Applied Analysis, vol. 2013, Article ID 260573, 7 pages, 2013.

[7] V. Soundararajan, H. Atharifar, and R. Kovacevic, "Monitorinf and processing the acoustic emission signals from the frictionstir-welding process," Proceedings of the Institution of Mechanical Engineers, Part B: Journal of Engineering Manufacture, vol. 220, no. 10, pp. 1673-1685, 2006.

[8] C. N. Suresha, B. M. Rajaprakash, and U. Sarala:, "Applicability of acoustic emission in the analysis of friction stir welded joints," International Journal of Recent Trends in Engineering, vol. 1, no. 5, pp. 86-89, 2009.
[9] A. S. Roca, H. C. Fals, E. J. Macías, J. B. Fernández, and F. S. Adán, "Time-frequency diagram applied to stability analysis in gas metal arc welding based on acoustic emission," Science and Technology of Welding and Joining, vol. 15, no. 3, pp. 226-232, 2010.

[10] E. J. Macías, A. S. Roca, H. C. Fals, J. B. Fernández, and M. P. de la Parte, "New stability index for short circuit transfer mode in GMAW process using acoustic emission signals," Science and Technology of Welding and Joining, vol. 12, no. 5, pp. 460-466, 2007.

[11] J. B. Fernández, A. S. Roca, H. C. Fals, E. J. Macias, and M. P. de la Parte, "Application of vibroacoustic signals to evaluate tools profile changes in friction stir welding on AA 1050 H24 alloy," Science and Technology of Welding and Joining, vol. 17, pp. 501510, 2012.

[12] J. X. Shen and W. Li, "Sensitivity analysis of wavelet neural network model for short-term traffic volume prediction," Journal of Applied Mathematics, vol. 2013, Article ID 953548, 10 pages, 2013.

[13] Y. D. Song, Q. Cao, X. Du, and H. R. Karimi, "Control strategy based on wavelet transform and neural network for hybrid power system," Journal of Applied Mathematics, vol. 2013, Article ID 375840, 8 pages, 2013.

[14] K. Dimililer, "Backpropagation neural network implementation for medical image compression," Journal of Applied Mathematics, vol. 2013, Article ID 453098, 8 pages, 2013.

[15] G. G. Wang, L. H. Guo, and H. Duan, "Wavelet neural network using multiple wavelet functions in target threat assessment," The Scientific World Journal, vol. 2013, Article ID 632437, 7 pages, 2013.

[16] Y. K. Yousif, K. M. Daws, and B. I. Kazem, "Prediction of friction stir Welding characteristic using neural network," Jordan Journal of Mechanical and Industrial Engineering, vol. 2, no. 3, pp. 151-155, 2008.

[17] E. J. Macías, A. S. Roca, H. C. Fals, J. B. Fernández, and J. C. S. Muro, "Neural networks and acoustic emission for modelling and characterization of the friction stir welding process," Revista Iberoamericana de Automática e Informática Industrial RIAI, vol. 10, no. 4, pp. 434-440, 2013.

[18] I. N. Tansel, M. Demetgul, H. Okuyucu, and A. Yapici, "Optimizations of friction stir welding of aluminum alloy by using genetically optimized neural network," International Journal of Advanced Manufacturing Technology, vol. 48, no. 1-4, pp. 95101,2010

[19] K. Lakshminarayanan and V. Balasubramanian, "Comparison of RSM with ANN in predicting tensile strength of friction stir welded AA7039 aluminium alloy joints," Transactions of Nonferrous Metals Society of China, vol. 19, no. 1, pp. 9-18, 2009.

[20] X. J. Yang, D. Baleanu, H. M. Srivastava, and J. A. T. Machado, "On local fractional continuous wavelet transform," Abstract and Applied Analysis, vol. 2013, Article ID 725416, 5 pages, 2013.

[21] W. Q. Song, Q. Li, Qing Li, and Y. M. Wang, "Tool wear detection using lipschitz exponent and harmonic wavelet," 
Mathematical Problems in Engineering, vol. 2013, Article ID 489261, 8 pages, 2013.

[22] C. Chen, R. Kovacevic, and D. Jandgric, "Wavelet transform analysis of acoustic emission in monitoring friction stir welding of 6061 aluminum," International Journal of Machine Tools and Manufacture, vol. 43, no. 13, pp. 1383-1390, 2003.

[23] M. S. Orozco, E. J. Macías, A. S. Roca, H. C. Fals, and J. B. Fernández, "Optimisation of friction-stir welding process using vibro-acoustic signal analysis," Science and Technology of Welding and Joining, vol. 18, no. 6, pp. 532-540, 2013. 


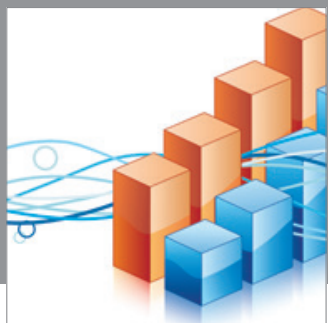

Advances in

Operations Research

mansans

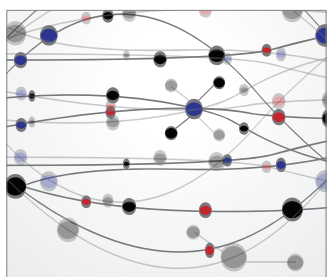

The Scientific World Journal
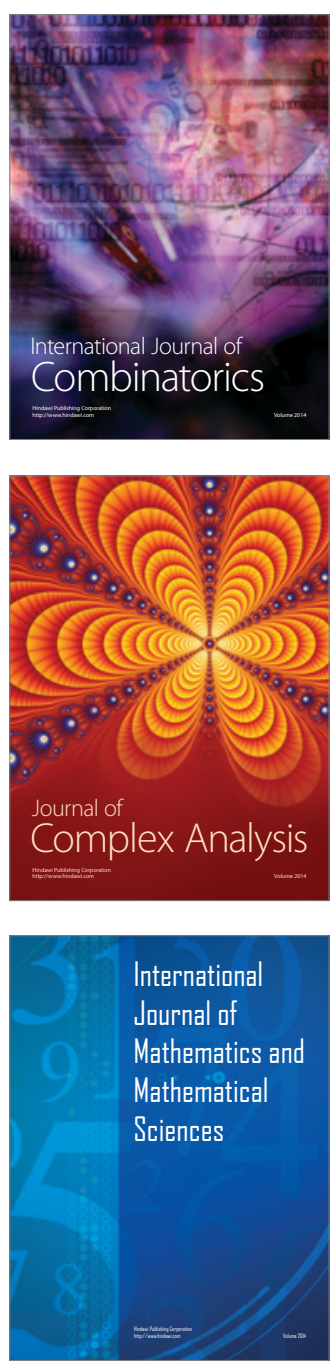
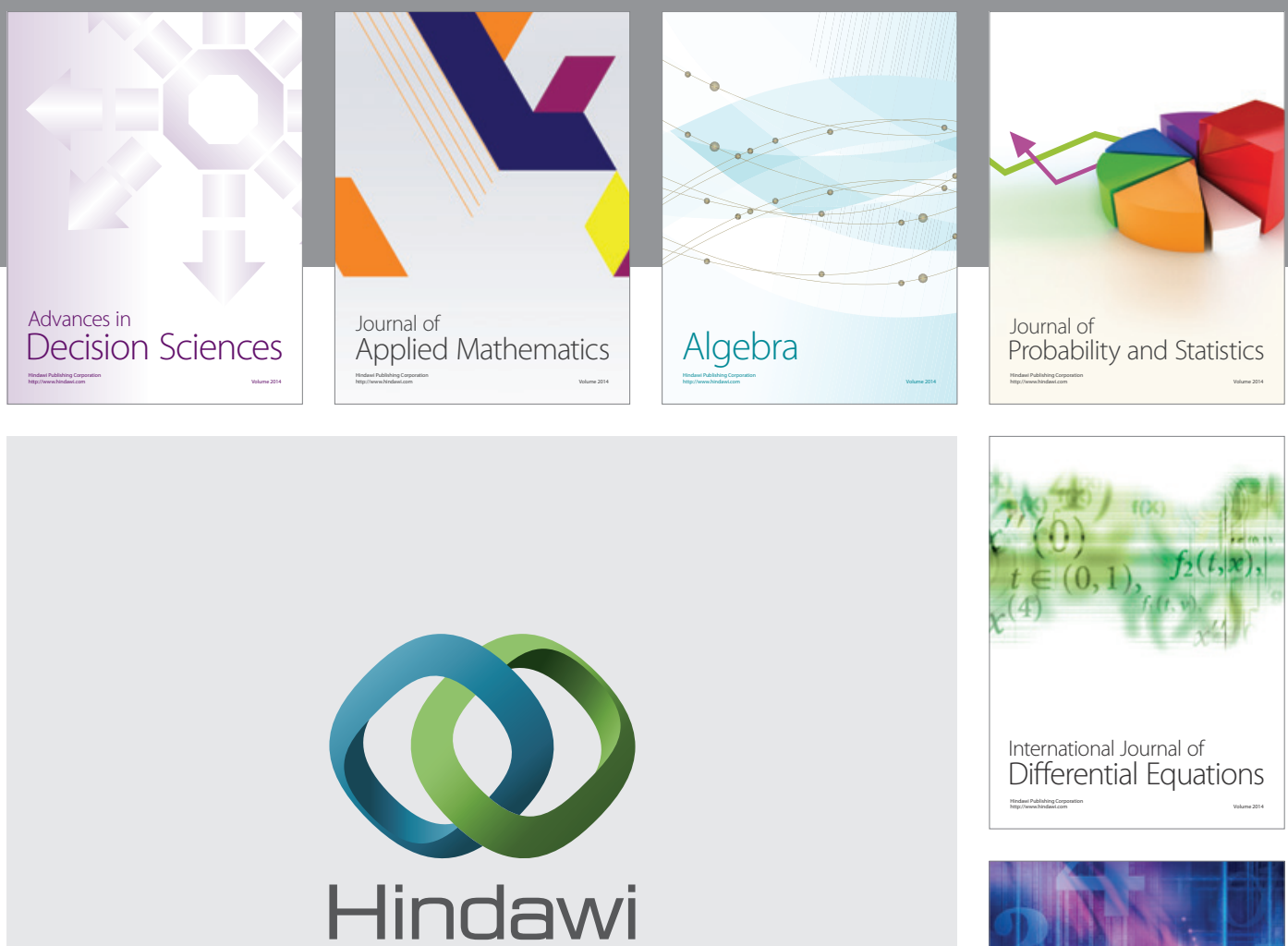

Submit your manuscripts at http://www.hindawi.com
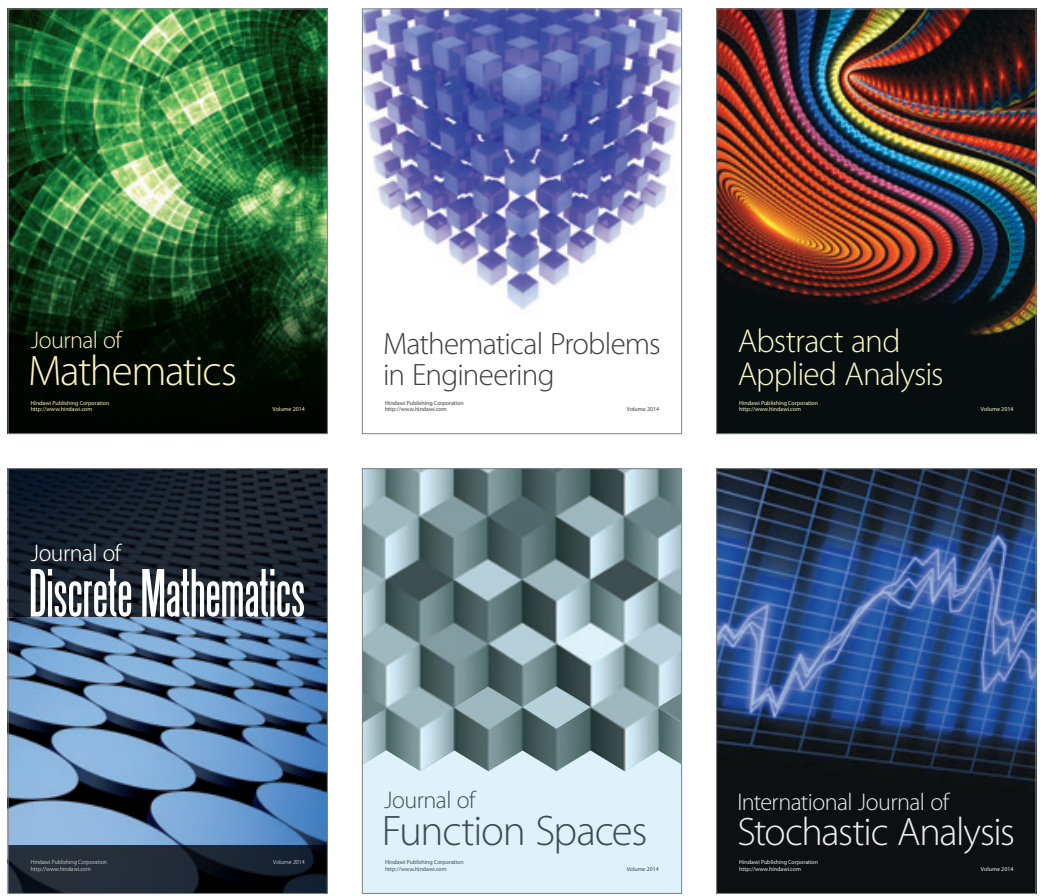

Journal of

Function Spaces

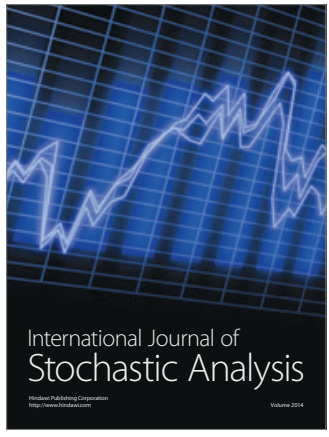

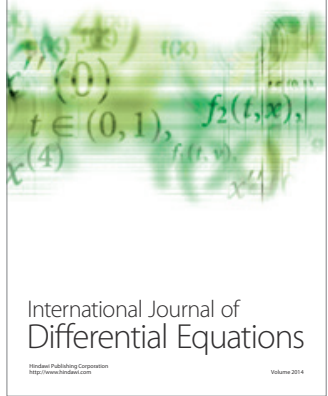
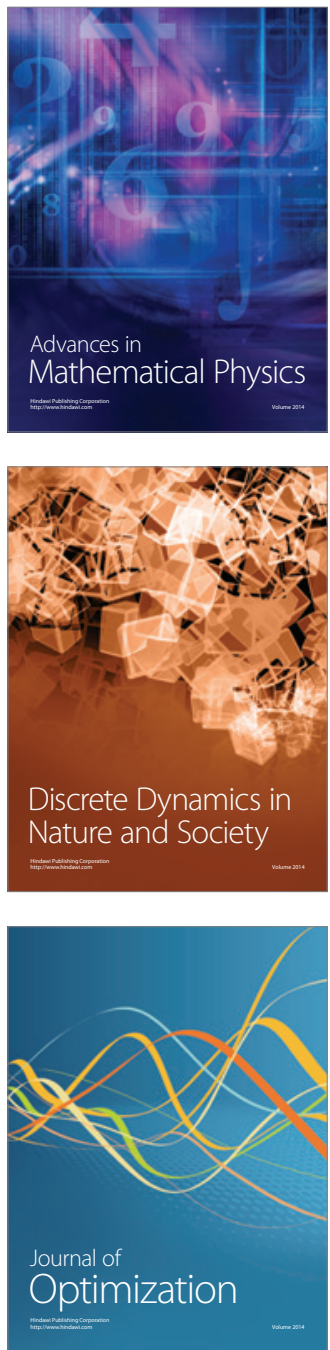